\title{
Festakt zu Ehren von Magdalene Schoch
}

\section{am 21. November 2012 in den Räumen der Universität Hamburg}

\author{
Magdalene Schoch hielt Abstand
}

\author{
Prof. Dr. Tilman Repgen \\ Dekan der Fakultät für Rechtswissenschaft, Universität Hamburg
}

Als Dekan der Fakultät für Rechtswissenschaft möchte ich Sie alle heute, am 21. November 2012, zum Festakt zu Ehren von Magdalene Schoch herzlich begrüßen. Wir freuen uns, dass Sie so zahlreich dieser Einladung zu einer Gedenkveranstaltung aus Anlass eines Ereignisses - nämlich des achtzigsten Jahrestags der Habilitation von Magdalene Schoch - gefolgt sind, das durch seine besonderen Umstände verdient, aus dem Alltag herausgehoben zu werden. Als Sie, Frau Lembke, als Initiatorin des heutigen Abends mir vor Monaten Ihre Idee vortrugen, rannten Sie gewissermaßen eine offene Tür ein. Ich danke Ihnen für diese Initiative und deren tatkräftige Umsetzung.

Im Wort „Gedenkveranstaltung“ steckt ein kürzeres Wort, zugegeben nicht eben gebräuchlich, das auf ihren Sinn hinweist: „Denk-veranstaltung“. Denken, Nachdenken - das ist hier gefragt. Die Geschichte von Magdalene Schoch möchte ich hier nicht erzählen. Darüber werden wir ja vor allem in dem Vortrag von Herrn Kollegen Nicolaysen hören, den ich zusammen mit Frau Kollegin Coester-Waltjen ebenfalls herzlich begrüßen möchte. Frau Coester-Waltjen wird das wissenschaftliche Werk Schochs würdigen. Die Geschichte Schochs bietet uns Stoff zum Nachdenken, einerseits natürlich über die Zeit damals, vor 80 Jahren, andererseits aber auch zum Nachdenken über das Heute, über uns, über unser Verständnis von Recht und Gerechtigkeit, Mut, Wissenschaft usw., also über Dinge, die uns heute angehen, auch wenn die Zeitumstände Gott sei Dank - andere geworden sind. Schon einfache Zahlen deuten auf gewichtige Unterschiede hin:

Schauen wir kurz auf die Fakultät 1932: Ihr gehören 13 Jura-Professoren an. Heute sind es 26 Lebenszeit-Professuren und elf Juniorprofessuren. Damals waren es 808 Studierende, heute zählen wir weit über 3.000. Unter den 808 Studierenden waren damals nur 65 Frauen, heute ist das Geschlechterverhältnis ausgewogen. Oder schauen wir kurz auf die Wirtschaftsdaten der damaligen Zeit: ${ }^{2}$

Zwischen 1928 und 1932 sank der deutsche Außenhandel der Menge nach auf 60 Prozent, dem Wert nach auf 38 Prozent. Trotz gestiegener Betriebskosten betrugen die Frachttarife 1932 nur noch 69 Prozent des Standes von 1913. Die Werft Blohm und Voss verringerte ihren Personalbestand von 10.700 Beschäftigten im Jahre 1929 auf 2.449 Ende 1932. In Hamburg verringerte sich der Umsatz zwischen 1928 und 1932 um 50 Prozent. Gab es 1928 in Hamburg 50.000 Erwerbslose, waren es 1930 schon 100.000 und 1932 165.000. Die erst 1927 eingeführte Arbeitslosenpflichtversicherung konnte die Erwerbslosen nicht vor der Verelendung bewahren. Durchschnittlich erhielt ein lediger Hamburger Erwerbsloser 37 Prozent seines letzten Einkommens als Unterstützung. Dauerte die Arbeitslosigkeit über ein Dreivierteljahr, so blieb nur die Wohlfahrtshilfe, die kaum genügte, um die Versorgung mit Grundnahrungsmitteln sicherzustellen. Die Arbeitslosenversicherung war auf Siebenhundert- bis Achthunderttausend Arbeitslose berechnet, 1930 zählte man aber über drei Millionen Erwerbslose, 1931 4,5 Millionen, 1932 mehr als 5,6 Millionen. Die industrielle Produktion Deutschlands sank 1932 auf ca. 60 Prozent des Standes von 1928. ${ }^{3}$ In Hamburg bedeutete das zum Beispiel, dass 1932 rund 22 Prozent der Gesamtbevölkerung von der Wohlfahrtshilfe leben musste. ${ }^{4}$ Auch diejenigen, die noch Arbeit hatten, mussten in der Regel Lohn- oder Gehaltskürzungen von nominell ca. 30 Prozent hinnehmen - das galt - heute schwer vorstellbar auch für die Beamten und Angestellten im öffentlichen Dienst. Zwar gab es gleichzeitig auch einen Preisverfall (Deflation), aber der Kaufkraftschwund überstieg diesen deutlich.

Blickt man auf das Rechtssystem, so fallen Gemeinsamkeiten auf: Das BGB galt schon damals - wenngleich heute vielfach verändert. Die Zivilprozessordnung galt schon damals - wenngleich heute vielfach verändert. Und ich könnte manche Gesetze aufzählen, die uns mit dieser Zeit verbinden. Wie stand es aber um das Denken über Recht und Unrecht? Auch hier lassen sich - bezogen auf das Jahr 1932 - viele Gemeinsamkeiten finden.

Die Biografie von Magdalene $S c h o c h^{5}$ regt aber zum Nachdenken über Recht und Unrecht an, gerade vor dem Hintergrund der Entwicklungen seit 1933, die in unvorstellbare Verbrechen, insbesondere in die Shoa mündeten.

Dieses Nachdenken anhand historischer Ereignisse ist kein Selbstzweck, auch wenn es in sich selbstverständlich als wissenschaftliches Anliegen völlig legitim ist.

Aristoteles, der so vieles Gültiges zu unserem Denken beigetragen hat, hat einmal gemeint: „Wie überall, wird sich [...]

1 Begrüßung anlässlich des Festaktes der Fakultät für Rechtswissenschaft zu Ehren von Magdalene Schoch am 21.11.2012 in Hamburg. Die Form des Grußwortes ist beibehalten; die Anmerkungen beschränken sich auf die notwendigsten Nachweise.

2 Soweit nicht anders angegeben, sind die Zahlen Büttner, Ursula, Der Stadtstaat als demokratische Republik, in: Hamburg. Geschichte einer Stadt, Band 2, hrsg. von Werner Jochmann, Hamburg 1986, S. 131 ff., hier S. 240-244, entnommen.

3 Marcowitz, Reiner, Weimarer Republik 1929-1933, 3. Auflage, Darmstadt 2009, S. 20 f.

4 Ebd., S. 21.

5 Dazu: Nicolaysen, Rainer, Konsequent widerstanden - die Juristin Magdalene Schoch, in: Das Hauptgebäude der Universität Hamburg als Gedächtnisort, Hamburg 2012, S. 171 ff. Dieser Darstellung sind alle biografischen Fakten im Folgenden entnommen. 
die rechte Einsicht erst ergeben, wenn man die Dinge sich von ihren Ursprüngen her entwickeln sieht. " ${ }^{6}$ Es geht um Einsicht. Hier haben wir die Chance, unsere Einsicht in den Wert des Rechts und des Anstands, aber auch in menschliche Abgründe zu schärfen.

Magdalene Schoch beeindruckt heute vor allem durch ihre klare Einsicht in das Unrecht, das der Nationalsozialismus von Anfang an gebracht hat, insbesondere gegenüber den Juden. Konsequent hielt sie weiter Kontakt zu jüdischen Wissenschaftlern. Ihre 1934 publizierte Habilitationsschrift widmete sie dem 1933 entlassenen und nach England emigrierten Lehrer Albrecht Mendelssohn Bartholdy. Ungeachtet der Warnungen des Hamburger Rektors vor beruflichen Nachteilen nahm sie als einziges Mitglied der Hamburger Fakultät an der Beerdigung ihres Lehrers 1936 in Oxford teil. 1937 weigerte sie sich, der Aufforderung zum Eintritt in die NSDAP zu folgen. Im Sommer desselben Jahres kündigte sie ihre Stelle in Hamburg und emigrierte wenig später in die Vereinigten Staaten, ohne bereits eine Anstellung zur Finanzierung des Lebensunterhalts sicher zu haben.

In der historischen Wissenschaft hat sich ein sehr differenzierter Umgang mit dem Begriff des Widerstands entwickelt.? Soll man den punktuellen Loyalitätsentzug bereits als Widerstand bezeichnen - oder nur die politische Aktivität, die auf Umsturz zielt? Neben dem Begriff des Widerstands hat sich derjenige des „Abstands“ etabliert. Das passt, wie mir scheint, auch zu Magdalene Schoch. Sie selbst verstand sich wohl nicht als Widerständlerin. Sie sagte von sich, „ein Leben, das unerträglich geworden ist, aufzugeben und ein neues Leben zu suchen, [sei] nichts weiter als Selbsterhaltungstrieb ...". Aber sie hat doch konsequent und mutig Abstand gehalten - Leo Raape hatte das schon damals erkannt. ${ }^{8}$ Sie war angesichts des Unrechts bereit, auf ihre berufliche Laufbahn in Deutschland zu verzichten. Ein Unrecht der Missachtung, der Diskriminierung, der Entrechtung, das auch in unserer Fakultät geschah. Schoch wollte sich an all' dem nicht nur nicht beteiligen, sondern sie forderte die Achtung der Rechte, den Anstand im Umgang miteinander ein. Darüber werden wir heute noch mehr hören.

Vor dem Hintergrund der Einsicht in die geschichtlichen Zusammenhänge erscheint es mir angebracht, im Namen unserer Fakultät unser Bedauern darüber auszudrücken, dass Magdalene Schoch und so vielen anderen in unserer Fakultät Unrecht geschehen ist. Wir können heute nicht mehr Wiedergutmachung leisten, aber wir können um Vergebung bitten und genau das möchte ich für unsere Institution tun.

6 Zitiert nach: Jaeger, Werner, Aristoteles. Grundlegung einer Geschichte seiner Entwicklung, 2. Auflage, Berlin 1955, S. 2.

7 Dazu zusammenfassend: Repgen, Konrad, Widerstand oder Abstand? Kirche und Katholiken in Deutschland 1933 bis 1945, in: Geschichtswissenschaft und Zeiterkenntnis. Von der Aufklärung bis zur Gegenwart. Festschrift zum 65. Geburtstag von Horst Möller, hrsg. von Klaus Hildebrand, Udo Wengst und Andreas Wirsching, München 2008, S. 555 ff.

8 Das Zitat von Magdalene Schoch und die Äußerung Leo Raapes stammen aus einem Brief von Schoch an ihre Mutter aus dem Sommer 1938, hier zitiert nach: Nicolaysen, Rainer, Konsequent widerstanden - die Juristin Magdalene Schoch, in: Das Hauptgebäude der Universität Hamburg als Gedächtnisort, Hamburg 2012, S. 171 (182)

\section{Dank, Rückblick und Ausblick'}

\section{Jun.-Prof. Dr. Ulrike Lembke}

Juniorprofessur für Öffentliches Recht und Legal Gender Studies, Universität Hamburg

Meine Damen und Herren, liebe Kolleginnen und Kollegen, liebe Studierende,

ich weiß, Sie warten ungeduldig auf Festrede und Festvortrag, aber gestatten Sie mir zuvor einige - eher persönliche Worte.

Zunächst einmal darf ich mich bedanken. Ich bedanke mich sehr gern, denn es hat mich gerührt, wie viel Zuwendung und Unterstützung die Idee des Festaktes erfahren hat, nachdem sie einmal geboren war. (Solch absolut ungeteilte Zustimmung zu einem „Gender-Projekt“ war für mich eine neue Erfahrung, die ich sehr genossen habe!) Dass die Idee zu einem Festakt für Magdalene Schoch allerdings überhaupt entstehen konnte, verdanken wir unseren Studierenden. Sie waren es, die mich nach einer Vorlesung gefragt haben, wie die Frau eigentlich hieß, die sich als erste Juristin in Deutschland habilitiert hat, und an welcher Universität dieses denkwürdige Ereignis stattfand. Ich wusste es nicht. Die Recherche mit Hilfe des Juristinnen-Lexikons ${ }^{2}$ förderte Überraschendes zutage: Magdalene Schoch hatte sich 1932 an der Hamburger Rechts- und Staatswissenschaftlichen Fakultät habilitiert! Und mit Blick auf die Jahreszahl stand unverzüglich fest, dass dieses Ereignis gefeiert werden musste.

Dass aus der Vermehrung historischer Kenntnisse und der daraus geborenen Idee in so knapp bemessener Zeit dieser Festakt entstehen konnte, verdankt sich der vorbehaltlosen Unterstützung vieler Personen, denen ich zu größtem Dank verpflichtet bin. Zunächst möchte ich mich bei Prof. Dr. Dagmar Coester-Waltien und Prof. Dr. Rainer Nicolaysen bedanken, die ohne Zögern die Festvorträge zugesagt und damit den inhaltlichen Erfolg dieser Veranstaltung gesichert haben. Herr Nicolaysen hat überdies alle seine Unterlagen über Magdalene

1 Grußwort anlässlich des Festaktes der Fakultät für Rechtswissenschaft zu Ehren von Magdalene Schoch am 21.11.2012 in Hamburg. 2 Röwekamp, Marion, Juristinnen - Lexikon zu Leben und Werk, Baden-Baden 2005, hrsg. vom Deutschen Juristinnenbund e.V. 